\title{
Modelling chaos in asymmetric optical fibres
}

\author{
D. S. Kumar' ${ }^{1}$, S. Creagh ${ }^{2}$, S. Sujecki ${ }^{1}$, T. M. Benson ${ }^{1}$ \\ ${ }^{1}$ George Green Institute for Electromagnetics Research, Faculty of Engineering \\ ${ }^{2}$ School of Mathematical Sciences \\ University of Nottingham, University Park, Nottingham NG7 2RD, UK \\ Tel: +44 (0) 115951 5589, e-mail: trevor.benson@nottingham.ac.uk
}

\begin{abstract}
A ray dynamical approach is developed for the study of large-core asymmetric step index fibres (SIF), especially those made from chalcogenide glasses (ChGs) which can exhibit very high refractive index, large numerical aperture, and which are transparent at mid-infrared wavelengths. The model allows for deformations of the SIF away from concentric circular structures, and for the light rays captured by the fibre to behave chaotically within the asymmetric boundaries of the fibre. Chaotic and periodic rays can be classified by the Poincare surface of sections (SOSs). In the model, the ray dynamics in the SIF are approximated by dividing the SOSs into pixels; the construction of a transfer matrix stores all the mapping probabilities. The light intensity distribution in the SOSs is efficiently propagated using the constructed transfer matrix, providing a viable alternative to propagating all the rays in the SIF by brute force ray tracing. The model enables the rapid calculation of the power accumulated in the fibre core following an arbitrary excitation.
\end{abstract}

Keywords: Surface of sections, chaos, chalcogenide glasses, mid-infrared photonics, step index fibre.

\section{INTRODUCTION}

Bright fibre-based mid-IR light sources for the mid-infrared (MIR) region (3 25 $\mu m$ wavelength) are being intensively researched, driven by many potential applications that include medical diagnostics and environmental monitoring [1]. Chalcogenide glasses (ChGs) are promising host materials for optical operations in the MIR region $[1,2]$. Prior work includes the successful fibre-drawing of low optical loss, optically clad, lanthanide ion doped GeAsGaSe core/GeAsGaSe cladding chalcogenide fibre as a crucial milestone towards the realisation of fibre lasers in the mid-IR [3]. The ChGs have a large refractive index, typically in the range from 2 to 4 depending on composition, enabling the realisation of step index fibres (SIF) with exceptionally high numerical aperture and acceptance angles [4]. However, the fabrication SIF with a circular and coaxial core using a rod in tube and/or coextrusion process is challenging due to the different physical glass properties, alignment issues, and the uneven pressure during preform extrusion. The geometry of fabricated SIF can often become asymmetric and light guided through the fibre is then expected to show chaotic behaviour, as shown in fig. 1(a). As noted by Leproux et al., [5], and encouraging the studies reported in the present contribution, this deformation and the accompanying chaotic propagation can be beneficial, for example in the design of double-clad fibre amplifiers. Thus, one may well consider deliberately introducing such geometrical distortions.

The standard way to classify rays as either chaotic or periodic is to depict them in a Poincare surface of sections (SOSs). It is a 2D phase space where the vertical axis shows the momentum and the horizontal axis shows the position of the rays. In the case of the SIF, the rays are only registered when they hit the boundaries. The length of the arc $s$ along the boundary gives the position of the ray, and the sine of the incident angle $\chi_{i}$ times the refractive index of the medium $n$ gives the momentum of the ray (denoted by $\mathrm{P}$, where $P=n \sin \chi_{i}$ ) as shown in fig. 1(b).

The SOSs for each boundary of the SIF can be divided into pixels. The idea of pixelating the phase space was discussed by Kullig and Wiersig in the context of microdisk cavities [6]; the focus of the present paper is the dynamics of rays inside a SIF rather than in an asymmetric resonant cavity although the discussion herein is restricted to two-dimensions. Each pixel contains a fraction of the total power in the whole phase space, this normalised power is redistributed in the SOS after each reflection. At the dielectric boundary between core and cladding, Snell's law conserves the momentum of the rays being transmitted and reflected. Thus,

$$
n_{1} \sin \chi_{1}=n_{2} \sin \chi_{2}
$$

where a ray is refracted at the interface of the dielectric medium 1 and 2. 


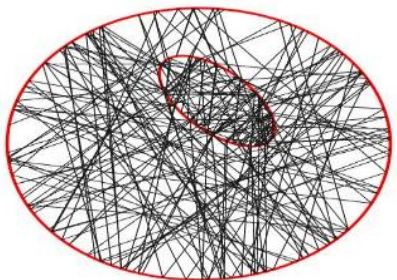

(a)

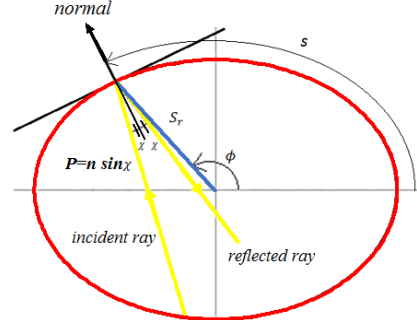

(b)

Fig. 1: (a) Cross-section of the SIF showing chaotic light rays. The core and cladding boundaries are both deformed from a circular shape, and are not co-axial, resulting in an asymmetric geometry. The rays bouncing around the fibre are a mixture of periodic and chaotic motions, and the classification can be made by illustrating the ray dynamics in the Poincaré surface of sections (SOSs). (b) The arc length $s$ forms the horizontal axis, and $P=n \sin \chi$ forms the vertical axis of the SOS. Each boundary in the step index fibre (SIF) cross-section has its own SOS.

The mapping of intensity from one pixel to others can be found by launching a bundle of rays inside one pixel and tracing them until they reach other positions within the boundary. The mapping information for all the pixels can be stored in the form of a matrix that transfers power from a set of pixels to the next for each reflection. This matrix is termed the transfer matrix or $\mathrm{T}$ matrix, and this approach efficiently approximates the ray tracing dynamics inside the fibre, where the number of rays to be traced would increase exponentially due to ray splitting at the dielectric boundaries after each reflection in a conventional approach. Tracing the ever-growing rays in the fibre individually would require an enormous computational effort, soon rendering the direct approach intractable.

\section{THE TRANSFER MATRIX APPROACH}

The T matrix is formed by dividing the SOS into pixels. The cladding boundary of the SIF is presently assumed to be perfectly reflecting. Since the ChGs have a high refractive index compared to that of air, this assumption is a reasonable one for most beams captured by the fibre input facet. Figure 2 shows the procedure of calculating the mapping the intensity from one pixel to others for 1 reflection. A bundle of equally placed rays is launched from the blue-shaded pixel in fig. 2 (a) and traced numerically until they reflect at the boundaries. Figure 2(b) shows the traced rays that have distributed themselves over multiple pixels after a reflection. The intensity in each pixel is calculated by finding the fraction of the total rays that have landed on it. The calculation shown in fig. 2 is performed for a single boundary SOS which has a deformation.

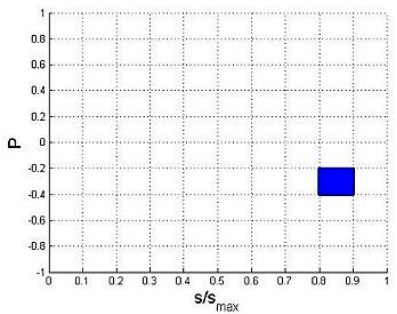

(a)

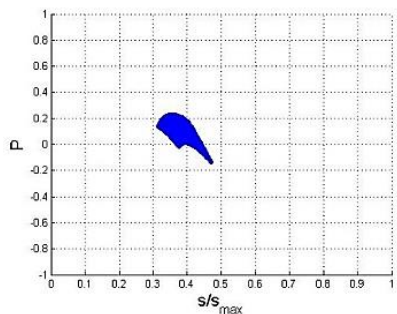

(b)

Fig. 2: Poincaré SOS divided into pixels and the intensity distributed from a pixel is mapped by tracing a bundle of rays. (a) The starting distribution is confined in this illustration to an individual pixel. (b) The intensity is distributed over multiple pixels after the ray bundle is traced for 1 reflection.

\subsection{Propagation of intensity along the fibre}

The procedure outlined above is now extended to the $2 \mathrm{D}$ cross-section of a SIF, such as that shown in fig. 1(a). Any input intensity distribution captured by the fibre, for example the Gaussian distribution illustrated in Fig. 3, can be mapped on to the pixelated SOS and subsequently propagated by the T matrix as it reflects along the fibre boundaries. The core refractive index $n_{c}$, taken here as 2.7 , is higher than that of the cladding, $n_{c l}$ taken here as 1.5, and has an outward facing and an inward facing boundary. The outer boundary SOS of the core registers any light from the cladding that reflects off the core and any light from the core that has transmitted out into the cladding. The inward facing, or inner, core boundary SOS registers any light from the cladding that has transmitted into the core and light from the core that has partially or total internally reflected. The Gaussian intensity distribution of fig. 3 is within the cladding SOS; the intensity is in the positive half of the $P$ axis but spread across the cladding arc length. Figure 4 shows the propagation of the initial Gaussian intensity distribution calculated by the T matrix after 1, 5 and 10 reflections. For comparison, the same distribution was ray traced up to 10 reflections as shown in fig. 5 . 


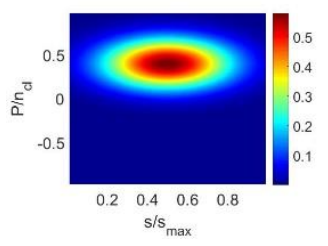

Fig. 3: An initial Gaussian intensity distribution at the cladding SOS used for propagation along the fibre using the T matrix. Both axes of the SOS have been normalised ( $n_{c l}$ is the refractive index of the cladding).

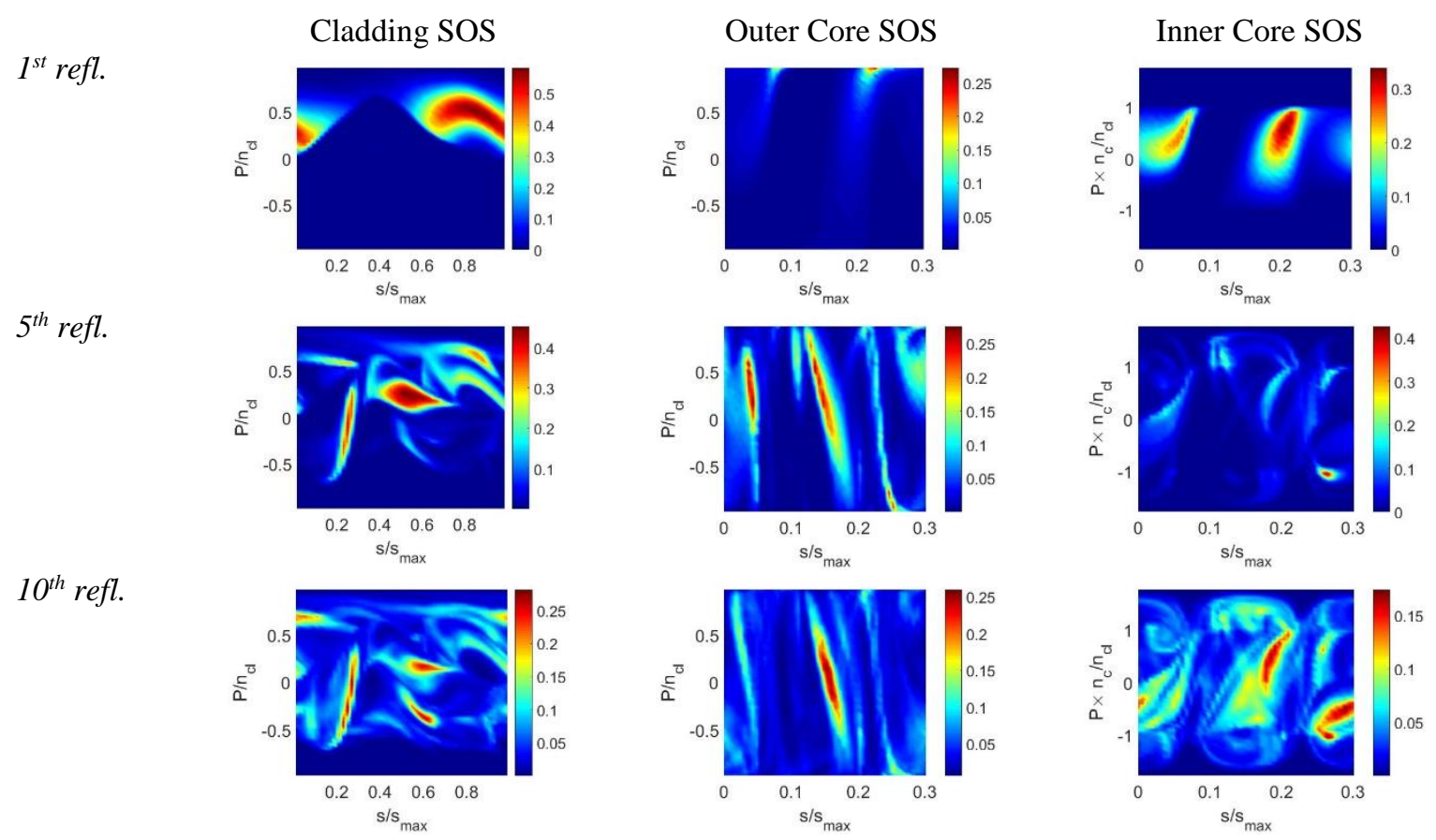

Fig. 4: Intensity distribution in the SOSs of the cladding, outer, and inner core boundaries formed by propagating the Gaussian initial distribution of fig. 3 by the T matrix $\left(n_{c}\right.$ is the refractive index of the core).

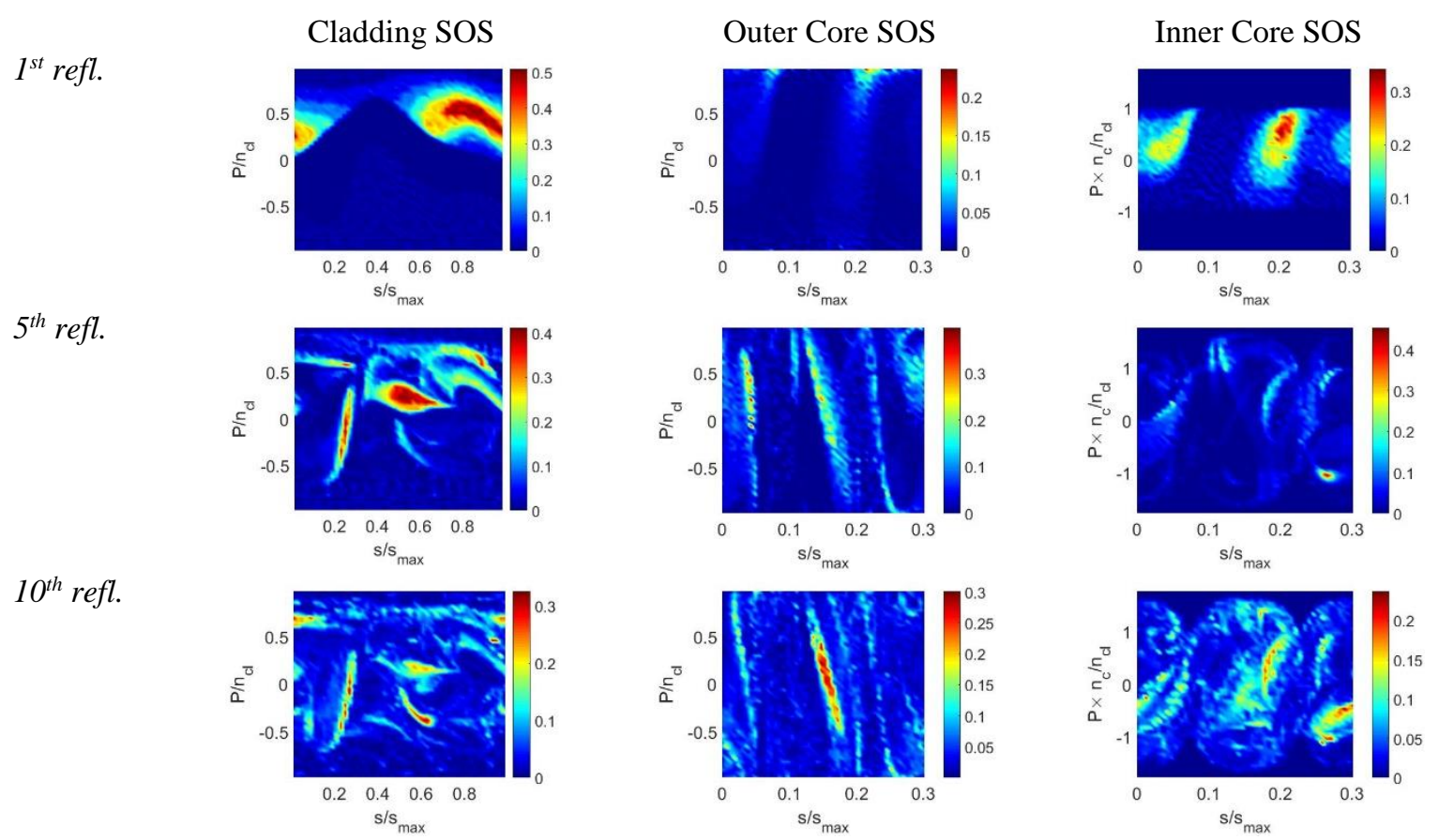

Fig. 5: Intensity distribution in the SOSs produced by ray tracing for up to 10 reflections. This compares well with the distribution produced by T matrix propagation as shown in fig.4. 


\subsection{Quantitative comparison between the $T$ matrix approach and ray tracing}

It was shown in section 2.1 that the $\mathrm{T}$ matrix approach closely approximates the behaviour of intensities distributed by ray tracing. To have a measure of this approximation, the SOSs can be divided into broad regions and the amount of power in those regions can be compared between the two methods. A convention was followed where the rays travelling in counter-clockwise (CCW) manner were considered having positive momenta. Thus, the positive half of the SOS represents the intensities of light reflecting in $\mathrm{CCW}$ manner around the fibre. Intensities of rays in the SOSs having positive and negative momenta are compared between the two methods after 10 reflections in fig. 6 .
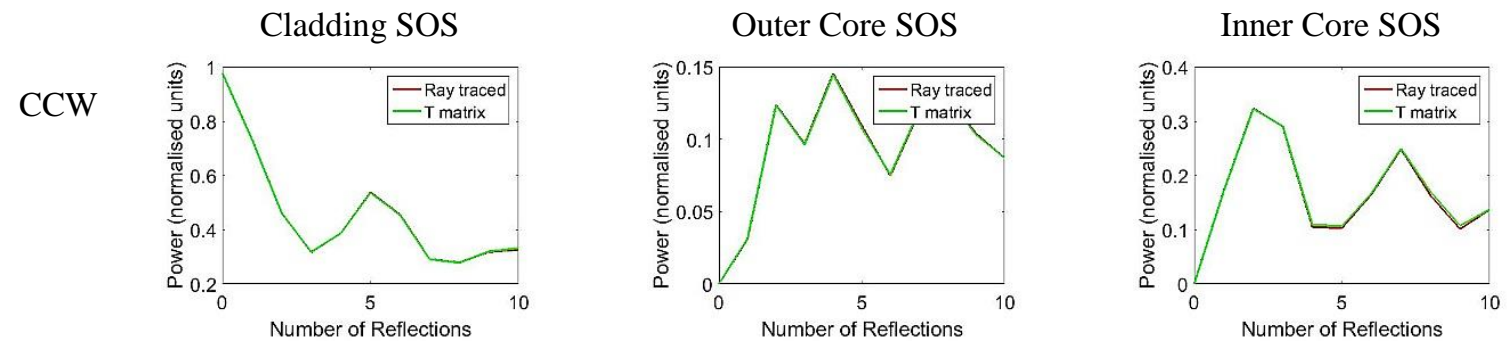

$\mathrm{CW}$
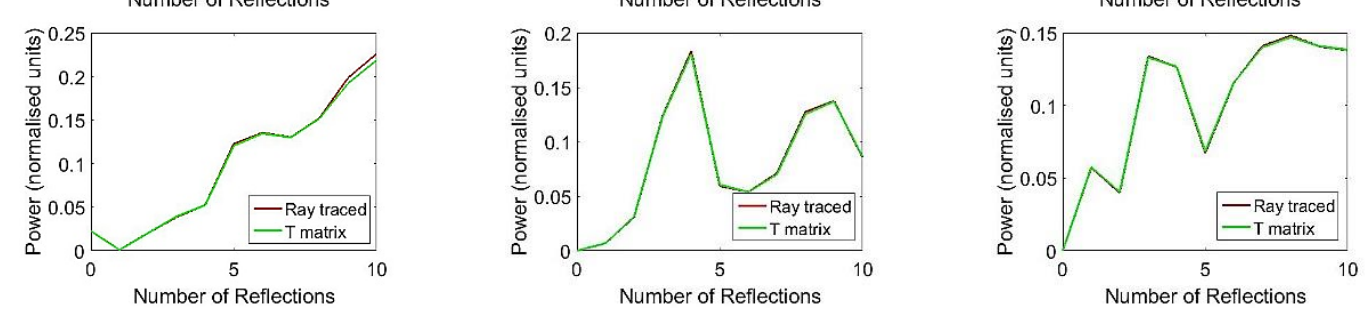

Fig. 6: Power in the propagated rays having counter-clockwise $(C C W)$ and clockwise $(C W)$ momenta, as calculated using the T matrix and ray tracing methods, in the cladding, outer and inner core SOSs. The powers calculated by the two methods show such a good agreement that the two curves are virtually indistinguishable in all plots.

\subsection{Net power delivered to the core}

Whilst direct ray tracing in due course becomes impracticable, owing to the rapid increase in the number of rays to be traced, with the T matrix approach it is possible to monitor the distribution until it reaches a steady state after many more reflections, and to calculate the net flow of power into the core. It is emphasised that once the T matrix is formed, many reflections can be simulated with little extra computational effort. Figure 7 shows the net accumulated power in the core that occurred due to the initial Gaussian intensity distribution, which was calculated by registering the differences between power flows in and out of the core after each reflection. The steady state was reached after about 200 reflections.

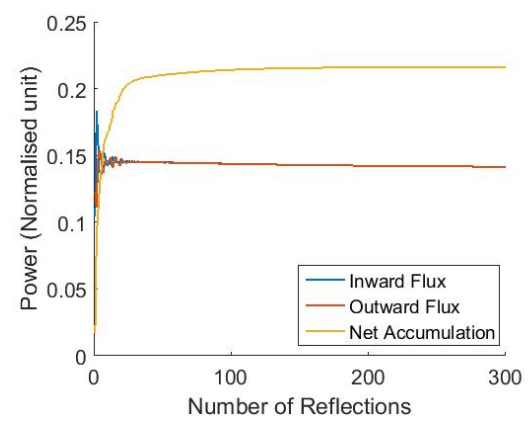

Figure 7: Net power accumulated in the core due to excitation of the cladding SOS by the Gaussian distribution of fig. 4. Steady state is reached after 200 reflections where the power going into the core (inward flux) became equal to the power going out of the core (outward flux).

\section{CONCLUSION}

The $\mathrm{T}$ matrix approach provides an efficient way to calculate the intensity distribution within a large core SIF in the presence of fibre deformation and a dielectric contrast. The deformation in the fibre introduces chaotic ray behaviour in the system, and the dielectric interface between the core and cladding causes ray splitting. Hence 
direct ray tracing rapidly becomes intractable, whereas the T matrix approach can propagate the rays for many reflections. The matrix approach also enables the modelling of the fibre for optimal launch conditions at the input, and maximising power captured by the core. The model appears to be especially promising for modelling SIFs based on chalcogenide glasses including those with an active core with gain offering signal amplification.

\section{ACKNOWLEDGEMENT}

DSK acknowledges, with thanks, the financial support of his PhD studies received from the Faculty of Engineering (Dean's Scholarship) and School of Mathematical Sciences, University of Nottingham, UK.

\section{REFERENCES}

[1] A. Seddon, "A prospective for new mid-infrared medical endoscopy using chalcogenide glasses," International Journal of Applied Glass Science, vol. 2, no. 3, pp. 177-191, 2011.

[2] B. J. Eggleton, B. Luther-Davies and K. Richardson, "Chalcogenide photonics," Nature Photonics, vol. 5, no. 3, pp. 141-148, 2011

[3] L. Sójka, Z. Tang, D. Furniss, H. Sakr, A. Oladeji, E. Beres-Pawlik, H. Dantanarayana, E. Faber, A. Seddon, T. Benson and S. Sujecki, "Broadband, mid-infrared emission from Pr3+ doped GeAsGaSe and chalcogenide fiber, optically clad," Optical Matrerials, vol. 36, no. 6, pp. 1076-1082, 2014.

[4] H. G. Dantanarayana, N. Abdel-Moneim, Z. Tang, L. Sójka, S. Sujecki, D. Furniss, A. B. Seddon, I. Kubat, O. Bang and T. M. Benson, "Refractive index dispersion of chalcogenide glasses for ultra-high numericalaperture fiber for mid-infrared supercontinuum generation," Optical Materials Express, vol. 4, no. 7, pp. 1444-1455, 2014.

[5] P. Leproux, S. Février, V. Doya, P. Roy and D. Pagnoux, "Modeling and optimization of double-clad fiber amplifiers using chaotic propagation of the pump,” Optical Fiber Technology, vol. 6, pp. 324-339, 2001.

[6] J. Kullig and J. Wiersig, "Frobenius-Perron eigenstates in deformed microdisk cavities: Non-Hermitian physics and asymmetric backscattering in ray dynamics," New Journal of Physics, vol. 18, no. 1, p. 15005, 2016. 